\title{
THE CYCLOTOMIC POLYNOMIAL TOPOLOGICALLY
}

\author{
GREGG MUSIKER AND VICTOR REINER \\ To the memory of Mark Feshbach.
}

\begin{abstract}
We interpret the coefficients of the cyclotomic polynomial in terms of simplicial homology.
\end{abstract}

\section{INTRODUCTION}

This paper studies the cyclotomic polynomial $\Phi_{n}(x)$, which is defined as the minimal polynomial over $\mathbb{Q}$ for any primitive $n^{\text {th }}$ root of unity $\zeta$ in $\mathbb{C}$. It is monic, irreducible, and has degree given by the Euler phi function $\phi(n)$, with formula

$$
\Phi_{n}(x)=\prod_{j \in(\mathbb{Z} / n \mathbb{Z})^{\times}}\left(x-\zeta^{j}\right) .
$$

The equation

$$
x^{n}-1=\prod_{d \mid n} \Phi_{d}(x)
$$

gives a recurrence showing that all coefficients of $\Phi_{n}(x)$ lie in $\mathbb{Z}$.

Although well-studied, the coefficients of $\Phi_{n}(x)$ are mysterious [2, 10, 11, 15, 16, 18, 31. We offer here two interpretations for their magnitudes, as orders of cyclic groups. In the initial interpretation (Corollary 5 below) this group is a quotient of the free abelian group $\mathbb{Z}[\zeta]$ by a certain full rank sublattice.

The second interpretation is topological, given by Theorem 1 below, as the torsion in the homology of a certain simplicial complex associated with a squarefree integer $n=p_{1} \cdots p_{d}$. These simplicial complexes originally arose in the work of Bolker [6], reappeared in the work of Kalai [14] and Adin [1] on higher-dimensional matrix-tree theorems, and were shown to be connected with cyclotomic extensions in work of J. Martin and the second author [19. We review these simplicial complexes briefly here in order to state the result; see Section 4 for more details.

Given a positive integer $p$, let $K_{p}$ denote a 0 -dimensional abstract simplicial complex having $p$ vertices 1 , which we will label by the residues

$$
\{0 \bmod p, 1 \bmod p, \ldots,(p-1) \bmod p\}
$$

for reasons that will become clear in a moment.

2000 Mathematics Subject Classification. Primary 05B35; Secondary 11R18,55U10.

Key words and phrases. Cyclotomic polynomial, higher-dimensional tree, matroid duality, oriented matroid, simplicial matroid, simplicial homology.

First author supported by NSF Postdoctoral Fellowship and NSF grant DMS-1067183. Second author supported by NSF grant DMS-1001933.

${ }^{1}$ Note that here $K_{p}$ does not refer to a complete graph on $p$ vertices; we hope that this causes no confusion. 
Given primes $p_{1}, \ldots, p_{d}$, let

$$
K_{p_{1}, \ldots, p_{d}}:=K_{p_{1}} * \cdots * K_{p_{d}}
$$

be the simplicial join, [23, §62], of $K_{p_{1}}, \ldots, K_{p_{d}}$. This is a pure $(d-1)$-dimensional abstract simplicial complex, that may be thought of as the complete d-partite complex on vertex sets $K_{p_{1}}$ through $K_{p_{d}}$ of sizes $p_{1}, \ldots, p_{d}$.

The facets (maximal simplices) of $K_{p_{1}, \ldots, p_{d}}$ are labelled by sequences of residues $\left(j_{1} \bmod p_{1}, \ldots, j_{d} \bmod p_{d}\right)$. Denoting the squarefree product $p_{1} \cdots p_{d}$ by $n$, the Chinese Remainder Theorem isomorphism

$$
\mathbb{Z} / p_{1} \mathbb{Z} \times \cdots \times \mathbb{Z} / p_{d} \mathbb{Z} \stackrel{\Xi}{\longrightarrow} \mathbb{Z} / n \mathbb{Z}
$$

allows one to label such a facet by a residue $j \bmod n$; call this facet $F_{j \bmod n}$. Then for any subset $A \subseteq\{0,1, \ldots, \phi(n)\}$, let $K_{A}$ denote the subcomplex of $K_{p_{1}, \ldots, p_{d}}$ which is generated by the facets $\left\{F_{j \bmod n}\right\}$ as $j$ runs through the following set of residues:

$$
A \cup\{\phi(n)+1, \phi(n)+2, \ldots, n-2, n-1\} .
$$

Our first main result interprets the magnitudes of the coefficients of $\Phi_{n}(x)$. Let $\tilde{H}_{i}(-; \mathbb{Z})$ denote reduced simplicial homology with coefficients in $\mathbb{Z}$.

Theorem 1. For a squarefree positive integer $n=p_{1} \cdots p_{d}$, with cyclotomic polynomial $\Phi_{n}(x)=\sum_{j=0}^{\phi(n)} c_{j} x^{j}$, one has

$$
\tilde{H}_{i}\left(K_{\{j\}} ; \mathbb{Z}\right)= \begin{cases}\mathbb{Z} / c_{j} \mathbb{Z} & \text { if } i=d-2, \\ \mathbb{Z} & \text { if both } i=d-1 \text { and } c_{j}=0, \\ 0 & \text { otherwise. }\end{cases}
$$

It should be noted that, starting with $d=3$ and for large enough primes $p_{i}$, one can exhibit $(d-1)$-dimensional subcomplexes of $K_{p_{1}, \ldots, p_{d}}$ with the following properties: like $K_{\{j\}}$, their only nonvanishing homology group lies in dimension $d-2$ and consists entirely of torsion, but unlike $K_{\{j\}}$, this torsion group need not be cyclic and can require arbitrarily many generators.

We furthermore interpret topologically the signs of the coefficients in $\Phi_{n}(x)$. For this, we use oriented simplicial homology, and orient the facet $F_{j \bmod n}$ having $j \equiv j_{i} \bmod p_{i}$ for $i=1,2, \ldots, d$ as

$$
\left[F_{j}\right]=\left[F_{j \bmod n}\right]=\left[j_{1} \bmod p_{1}, \ldots, j_{d} \bmod p_{d}\right] .
$$

Theorem 2. Fix a squarefree positive integer $n=p_{1} \cdots p_{d}$ with cyclotomic polynomial $\Phi_{n}(x)=\sum_{j=0}^{\phi(n)} c_{j} x^{j}$. Then for any $j \neq j^{\prime}$ such that $c_{j}, c_{j^{\prime}} \neq 0$, one has $\tilde{H}_{d-1}\left(K_{\left\{j, j^{\prime}\right\}} ; \mathbb{Z}\right) \cong \mathbb{Z}$, and any nonzero $(d-1)$-cycle $z=\sum_{\ell} b_{\ell}\left[F_{\ell}\right]$ in this homology group will have $b_{j}, b_{j^{\prime}} \neq 0$, with

$$
\frac{c_{j}}{c_{j^{\prime}}}=-\frac{b_{j^{\prime}}}{b_{j}} .
$$

In particular, $c_{j}, c_{j^{\prime}}$ have the same sign if and only if $b_{j}, b_{j^{\prime}}$ have opposite signs.

Example 3. We illustrate Theorems1 1 and2 for $n=15$. Here $d=2, p_{1}=3, p_{2}=5$, and $\phi(n)=2 \cdot 4=8$. The cyclotomic polynomial is

$$
\begin{aligned}
\Phi_{15}(x) & =1-x+x^{3}-x^{4}+x^{5}-x^{7}+x^{8} \\
& =(+1) \cdot\left(x^{0}+x^{3}+x^{5}+x^{8}\right)+(-1) \cdot\left(x^{1}+x^{4}+x^{7}\right)+0 \cdot\left(x^{2}+x^{6}\right) .
\end{aligned}
$$




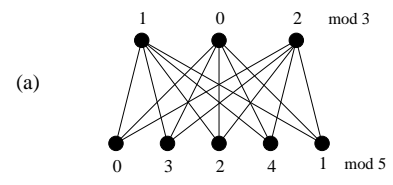

(b)

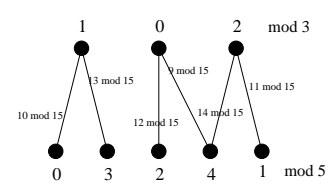

(c)

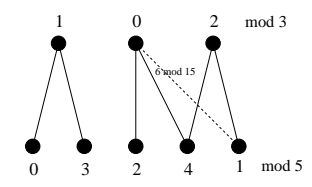

(d)

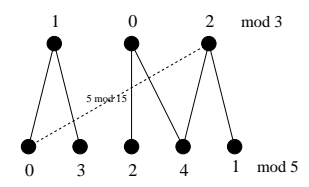

(e)

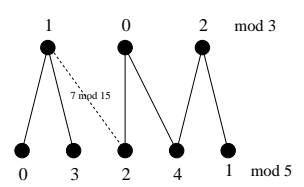

(f)
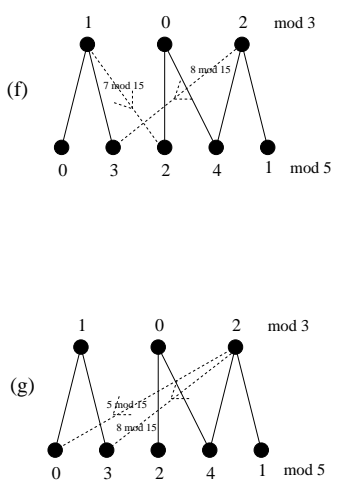

Figure 1. The case of $\Phi_{15}(x)$

The complex $K_{p_{1}, p_{2}}=K_{3,5}$ is a complete bipartite graph with vertex sets labelled as in Figure 1(a). The subcomplex $K_{\varnothing}$ generated by the edges $F_{j \bmod 15}$ with $j \in\{\phi(n)+1, \phi(n)+2, \ldots, n-1\}=\{9,10,11,12,13,14\}$ is the subgraph shown in Figure 1(b).

To see why the coefficient $c_{6}=0$ in $\Phi_{15}(x)$, one adds the edge $F_{6 \text { mod } 15}$ to the graph $K_{\varnothing}$, obtaining the graph $K_{\{6\}}$, shown in Figure 1(c), which has

$$
\begin{aligned}
& \tilde{H}_{0}\left(K_{\{6\}} ; \mathbb{Z}\right)=\mathbb{Z}=\mathbb{Z} / 0 \mathbb{Z} \\
& \tilde{H}_{1}\left(K_{\{6\}} ; \mathbb{Z}\right)=\mathbb{Z} .
\end{aligned}
$$

To see why the coefficients $c_{5}=+1$ or $c_{7}=-1$ have magnitude 1 , one adds the edge $F_{5 \bmod 15}$ or $F_{7 \bmod 15}$ to the graph $K_{\varnothing}$, obtaining the graphs $K_{\{5\}}$ or $K_{\{7\}}$ shown in Figures 1(d) and 1(e), which have

$$
\begin{aligned}
& \tilde{H}_{0}\left(K_{\{5\}} ; \mathbb{Z}\right)=0=\mathbb{Z} /(+1) \mathbb{Z} \\
& \tilde{H}_{0}\left(K_{\{7\}} ; \mathbb{Z}\right)=0=\mathbb{Z} /(-1) \mathbb{Z} .
\end{aligned}
$$

To understand the signs of the coefficients, note first that, by convention, $\Phi_{15}(x)$ is monic, so the coefficient $c_{8}=c_{\phi(n)}=+1$. Therefore any other coefficient $c_{j}$ should have sign

$$
\operatorname{sgn}\left(c_{j}\right)=\frac{\operatorname{sgn}\left(c_{j}\right)}{\operatorname{sgn}\left(c_{8}\right)}=-\frac{\operatorname{sgn}\left(b_{8}\right)}{\operatorname{sgn}\left(b_{j}\right)}
$$

where $z=\sum_{i} b_{i}\left[F_{i}\right]$ is a nontrivial cycle in $K_{\{j, 8\}}$, in which the edge $\left[F_{j}\right]$ is directed from the vertex $\left(j_{1} \bmod 3\right)$ toward the vertex $\left(j_{2} \bmod 5\right)$. As shown in Figures $1(\mathrm{f})$ and $1(\mathrm{~g})$, the nontrivial cycle in $K_{\{7,8\}}$ has $\left[F_{7}\right],\left[F_{8}\right]$ oriented in the same direction, explaining why $c_{7}=-1$, while the nontrivial cycle in $K_{\{5,8\}}$ has $\left[F_{5}\right],\left[F_{8}\right]$ oriented in the opposite direction, explaining why $c_{5}=+1$.

The remainder of the paper is structured as follows. Section2 describes an initial interpretation for the cyclotomic polynomial, which applies much more generally to any monic polynomial in $\mathbb{Z}[x]$. Section 3 reviews some facts, underlying the main results, about duality of matroids, Plücker coordinates, and oriented matroids. 
Section 4 recalls results and establishes terminology on Kalai's higher dimensional spanning trees in a simplicial complex. Section 5 discusses further properties of the simplicial complex $K_{p_{1}, \ldots, p_{d}}$ whose subcomplexes appear in Theorem 1 and 2 Section 6 prove these theorems. Section 7 gives some reformulations of Theorem 1 and 2 suggested to the authors by D. Fuchs. Section 8 explains how well-known properties of the cyclotomic polynomial manifest themselves topologically.

We remark that, since the submission of this paper for publication, an alternate proof of Theorem 1 has appeared in the work of R. Meshulam [21, using the Fourier transform on the group $\mathbb{Z}_{p_{1}} \times \cdots \times \mathbb{Z}_{p_{d}}$.

\section{Coefficients of MOnic polynomials in $\mathbb{Z}[x]$}

Our goal here is an initial interpretation for the coefficients of $\Phi_{n}(x)$, which applies more generally to the coefficients of any monic polynomial $f(x)$ in $\mathbb{Z}[x]$. Recall that when $f(x)$ is of degree $r$, one has an isomorphism of $\mathbb{Z}$-modules

$$
\begin{aligned}
\mathbb{Z}^{r} & \longmapsto \mathbb{Z}[x] /(f(x)) \\
\left(a_{0}, a_{1}, \ldots, a_{r-1}\right) & \longmapsto \sum_{j=0}^{r-1} a_{j} \bar{x}^{j} .
\end{aligned}
$$

As notation, let $R:=\mathbb{Z}[x] /(f(x))$, and for a subset $A$ of some abelian group, let $\mathbb{Z} A$ denote the collection of all $\mathbb{Z}$-linear combinations of elements of $A$.

Proposition 4. For a monic polynomial $f(x)=\sum_{j=0}^{r} c_{j} x^{j}$ of degree $r$ in $\mathbb{Z}[x]$, one has an isomorphism of abelian groups

$$
R / \mathbb{Z} A \cong \mathbb{Z} / c_{j} \mathbb{Z}
$$

where $A$ is the subset of size $r$ given as $\left\{\overline{1}, \bar{x}, \bar{x}^{2}, \ldots, \bar{x}^{r}\right\} \backslash\left\{\bar{x}^{j}\right\}$.

Proof. Consider the matrix in $\mathbb{Z}^{r \times(r+1)}$

$$
\left[\begin{array}{cccccc}
1 & 0 & 0 & \cdots & 0 & -c_{0} \\
0 & 1 & 0 & \cdots & 0 & -c_{1} \\
0 & 0 & 1 & \cdots & 0 & -c_{2} \\
\vdots & \vdots & \vdots & & \vdots & \vdots \\
0 & 0 & 0 & \cdots & 1 & -c_{r-1}
\end{array}\right]
$$

whose columns express the elements of $\left\{\overline{1}, \bar{x}, \bar{x}^{2}, \ldots, \bar{x}^{r}\right\}$ uniquely in the $\mathbb{Z}$-basis $\left\{\overline{1}, \bar{x}, \bar{x}^{2}, \ldots, \bar{x}^{r-1}\right\}$ for $R=\mathbb{Z}[x] /(f)$. The $r \times r$ submatrix obtained by restricting this matrix to the columns indexed by $A$ is equivalent by row and column operations (invertible over $\mathbb{Z})$ to a diagonal matrix with diagonal entries $\left(1,1, \ldots, 1,-c_{j}\right)$. Hence $R / \mathbb{Z} A \cong \mathbb{Z} / c_{j} \mathbb{Z}$.

The special case where $f(x)$ is the cyclotomic polynomial $\Phi_{n}(x)$ leads to the following considerations. Fix once and for all a primitive $n^{\text {th }}$ root of unity $\zeta$.

Corollary 5. The cyclotomic polynomial $\Phi_{n}(x)=\sum_{j=0}^{\phi(n)} c_{j} x^{j}$ has

$$
\mathbb{Z}[\zeta] / \mathbb{Z} A \cong \mathbb{Z} / c_{j} \mathbb{Z}
$$

where $A=\left\{1, \zeta, \zeta^{2}, \ldots, \zeta^{\phi(n)}\right\} \backslash\left\{\zeta^{j}\right\}$. 
Proof. Apply the previous proposition with $f(x)=\Phi_{n}(x)$ and $r=\phi(n)$, noting that the ring map $\mathbb{Z}[x] \rightarrow \mathbb{Z}[\zeta]$ sending $x$ to $\zeta$ will also send $x^{j}$ to $\zeta^{j}$, and induce an isomorphism $\mathbb{Z}[x] /\left(\Phi_{n}(x)\right) \rightarrow \mathbb{Z}[\zeta]$.

For later use (see the proof of Theorem 21), we prove here that the set

$$
P_{n}:=\left\{\zeta^{m}\right\}_{m \in(\mathbb{Z} / n \mathbb{Z})^{\times}}
$$

of all primitive $n^{\text {th }}$ roots of unity within $\mathbb{Z}[\zeta]$ forms a $\mathbb{Z}$-basis whenever $n$ is squarefree. This is a sharpening of an observation of Johnsen [13], who noted that $P_{n}$ forms a $\mathbb{Q}$-basis of $\mathbb{Q}[\zeta]$ in the same situation.

Proposition 6. When $n$ is squarefree, the collection $P_{n}$ of all primitive $n^{\text {th }}$ roots of unity forms a $\mathbb{Z}$-basis for $\mathbb{Z}[\zeta]$.

Proof. Note that $\left|P_{n}\right|=\phi(n)$ and that $\mathbb{Z}[\zeta]$ is a free abelian group of rank $\phi(n)$. Therefore it suffices to show that $P_{n}$ is a $\mathbb{Z}$-spanning set for $\mathbb{Z}[\zeta]$.

This spanning is clear when $n=p$ is prime since the only non-primitive root of unity is $1=-\left(\zeta+\zeta^{2}+\cdots+\zeta^{p-1}\right)$.

When $n=p_{1} \cdots p_{d}$ for $d>1$, temporarily use the notation $\zeta_{n}$ for a fixed primitive $n^{\text {th }}$ root of unity, and similarly for $\zeta_{p_{i}}$. The ring inclusions defined by

$$
\begin{aligned}
\mathbb{Z}\left[\zeta_{p_{i}}\right] \stackrel{f_{i}}{\longmapsto} \mathbb{Z}\left[\zeta_{n}\right] \\
\zeta_{p_{i}} \longmapsto\left(\zeta_{n}\right)^{\frac{n}{p_{i}}}
\end{aligned}
$$

assemble to give a $\mathbb{Z}$-module map $f:=f_{1} \otimes \cdots \otimes f_{d}$

$$
\begin{aligned}
\mathbb{Z}\left[\zeta_{p_{1}}\right] \otimes_{\mathbb{Z}} \cdots \otimes_{\mathbb{Z}} \mathbb{Z}\left[\zeta_{p_{d}}\right] & \longrightarrow \mathbb{Z}\left[\zeta_{n}\right] \\
\zeta_{p_{1}}^{a_{1}} \otimes \cdots \otimes \zeta_{p_{d}}^{a_{d}} & \longmapsto \zeta_{n}^{j}
\end{aligned}
$$

where $j \equiv \sum_{i=1}^{p} a_{i} \frac{n}{p_{i}} \bmod n$. Note that

$$
\begin{aligned}
\frac{n}{p_{i}} & \equiv 0 \bmod p_{i_{0}} \text { for } i \neq i_{0}, \\
\frac{n}{p_{i_{0}}} & \neq \equiv \bmod p_{i_{0}} .
\end{aligned}
$$

Consequently, for each $i_{0}=1,2, \ldots, d$, one has $j \equiv a_{i_{0}} \frac{n}{p_{i_{0}}} \bmod p_{i_{0}}$, and as $a_{i_{0}}$ runs over $\mathbb{Z} / p_{i_{0}} \mathbb{Z}$, the product $a_{i_{0}} \frac{n}{p_{i_{0}}}$ does the same. The map $f$ is then surjective by the Chinese Remainder Theorem isomorphism (2).

Lastly, note that the source of $f$ has a $\mathbb{Z}$-basis consisting of those $\zeta_{p_{1}}^{a_{1}} \otimes \cdots \otimes \zeta_{p_{d}}^{a_{d}}$ in which each $a_{i} \not \equiv 0 \bmod p_{i}$. Since this means that each $a_{i} \frac{n}{p_{i}} \not \equiv 0 \bmod p_{i}$, this basis maps under $f$ to the set $P_{n}$. Hence $P_{n}$ must $\mathbb{Z}$-linearly span the target $\mathbb{Z}[\zeta]$, and furthermore form a $\mathbb{Z}$-basis for the target.

\section{Duality of matroids or PlǘCKer Coordinates}

We will need a version of the linear algebraic duality between Plücker coordinates for complementary Grassmannians $G\left(r, \mathbb{F}^{n}\right), G\left(n-r, \mathbb{F}^{n}\right)$, or equivalently, the duality between bases and cobases in coordinatized matroids.

Proposition 7. Let $0 \leq r \leq n$. Let $M$ and $M^{\perp}$ be matrices in $\mathbb{F}^{r \times n}$ and $\mathbb{F}^{(n-r) \times n}$, respectively, both of maximal rank, with the following property: $\operatorname{ker} M$ is equal to the row space of $M^{\perp}$, or equivalently, $\operatorname{ker} M^{\perp}$ is the row space of $M$. Then 
(i) there exists a scalar $\alpha$ in $\mathbb{F}^{\times}$having the following property: for every $(n-r)$ subset $T$ of $[n]$, with complementary set $T^{c}$,

$$
\operatorname{det}\left(\left.M\right|_{T^{c}}\right)= \pm \alpha \cdot \operatorname{det}\left(\left.M^{\perp}\right|_{T}\right)
$$

where $\left.A\right|_{J}$ denotes the restriction of a matrix $A$ to the subset of columns indexed by $J$, and where the \pm sign depends upon the set $T$.

(ii) if one furthermore assumes that $\mathbb{F}=\mathbb{Q}$, that $M$ and $M^{\perp}$ have entries in $\mathbb{Z}$, and that there exists at least one $(n-r)$-subset $T_{0}$ for which $\left.M\right|_{T_{0}^{c}},\left.M^{\perp}\right|_{T_{0}}$ are both invertible over $\mathbb{Z}$, then the scalar $\alpha$ above equals \pm 1 , and one has for every $(n-r)$-subset $T$,

$$
\operatorname{coker}\left(\left.M\right|_{T^{c}}\right) \cong \operatorname{coker}\left(\left.M^{\perp}\right|_{T}\right) \text {. }
$$

Here we are regarding $\left.M\right|_{T^{c}}$ and $\left.M^{\perp}\right|_{T}$ as operators on $\mathbb{Z}^{r}$ and $\mathbb{Z}^{n-r}$, respectively.

Proof. For assertion (i), note that the hypotheses and conclusions are unchanged if one performs row operations over $\mathbb{F}$ separately on $M, M^{\perp}$, and if one permutes simultaneously the columns of $M, M^{\perp}$ by the same permutation of $[n]$.

Thus one can assume without loss of generality that the full rank matrix $M$ has the form $M=\left[I_{r} \mid A\right]$ for some matrix $A$ in $\mathbb{F}^{r \times(n-r)}$. In this case, the kernel of $M$ is spanned by the rows of $\left[-A^{t} \mid I_{n-r}\right]$, and hence by performing row operations on $M^{\perp}$, one can assume without loss of generality that $M^{\perp}=\left[-A^{t} \mid I_{n-r}\right]$.

When $M, M^{\perp}$ have these special forms, one has that

$$
\begin{aligned}
\left.M\right|_{T^{c}} & =\left[\begin{array}{lll}
\left.I_{r}\right|_{[r] \backslash T} & \mid & \left.A\right|_{[n] \backslash([r] \cup T)}
\end{array}\right] \\
\left.M^{\perp}\right|_{T} & =\left[\begin{array}{ll}
-\left.A^{t}\right|_{[r] \cap T} & \mid
\end{array}\right] .
\end{aligned}
$$

Hence det $\left(\left.M\right|_{T^{c}}\right)$ will be, up to sign, the determinant of $A$ restricted to its columns in $[n] \backslash([r] \cup T)$, and to its rows in $[r] \cap T$, while $\operatorname{det}\left(\left.M^{\perp}\right|_{T}\right)$ will be, up to sign, the determinant of $-A^{t}$ restricted to its columns in $[r] \cap T$, and to its rows in $[n] \backslash([r] \cup T)$. These determinants are the same up to sign.

For assertion (ii), again the hypotheses and conclusion are unchanged if one performs row operations invertible over $\mathbb{Z}$ to $M, M^{\perp}$, and if one permutes columns simultaneously in $M, M^{\perp}$. Thus without loss of generality, one can assume that

$$
\begin{aligned}
T^{c} & =[r] \\
T & =[n] \backslash[r] \\
M & =\left[I_{r} \mid A\right] \\
M^{\perp} & =\left[-A^{t} \mid I_{n-r}\right]
\end{aligned}
$$

as above. But in this case, one can check that coker $\left(\left.M\right|_{T^{c}}\right) \cong \operatorname{coker}\left(\left.M^{\perp}\right|_{T}\right)$ for the same reason that their determinants agree up to sign: their cokernels are isomorphic to the cokernels of matrices which are, up to sign, the transposes of each other.

The proof of Theorem 2 will ultimately rely on the following statement about duality of oriented matroids for vectors over an ordered field $\mathbb{F}$, such as $\mathbb{F}=\mathbb{Q}$.

Proposition 8. Let $\mathbb{F}$ be an ordered field, $M$ and let $M^{\perp}$ be matrices in $\mathbb{F}^{r \times n}$ and $\mathbb{F}^{(n-r) \times n}$ as in Proposition 7 , that is, both of maximal rank, with ker $M$ equal to 
the row space of $M^{\perp}$. Let the vectors $v_{\ell}$ in $\mathbb{F}^{r}$ and $v_{\ell}^{\perp}$ in $\mathbb{F}^{n-r}$ be the $\ell^{\text {th }}$ columns of $M$ and $M^{\perp}$.

Let $A$ be an $(r+1)$-subset of $\{1,2, \ldots, n\}$ such that the matrix $\left.M\right|_{A}$ in $\mathbb{F}^{r \times(r+1)}$ has full rank $r$, with

$$
\sum_{\ell \in A} c_{\ell} v_{\ell}=0
$$

the unique dependence among its columns, up to scaling.

Then for any pair of nonzero coefficients $c_{j}, c_{j^{\prime}} \neq 0$, the matrix $\left.M^{\perp}\right|_{A^{c} \cup\left\{j, j^{\prime}\right\}}$ in $\mathbb{F}^{(n-r) \times(n-r+1)}$ has full rank $n-r$, and the unique dependence among its columns, up to scaling,

$$
\sum_{\ell \in A^{c} \cup\left\{j, j^{\prime}\right\}} b_{\ell} v_{\ell}^{\perp}=0,
$$

will have both $b_{j}, b_{j^{\prime}} \neq 0$, with

$$
\frac{c_{j}}{c_{j^{\prime}}}=-\frac{b_{j^{\prime}}}{b_{j}} .
$$

In particular, $c_{j}, c_{j^{\prime}}$ have the same sign if and only if $b_{j}, b_{j^{\prime}}$ have opposite signs.

Proof. First let us show the assertion about ranks. Since $\left.M\right|_{A}$ has rank $r$, the fact that $c_{j^{\prime}} \neq 0$ implies that $v_{j^{\prime}}$ lies in the span of the columns of $\left.M\right|_{A \backslash\left\{j^{\prime}\right\}}$. Hence the matrix $\left.M\right|_{A \backslash\left\{j^{\prime}\right\}}$ in $\mathbb{F}^{r \times r}$ has rank $r$, and its columns give a basis for $\mathbb{F}^{r}$. Then Proposition [7(i) implies that the columns of $\left.M^{\perp}\right|_{A^{c} \cup\left\{j^{\prime}\right\}}$ form a basis of $\mathbb{F}^{n-r}$, and thus $\left.M^{\perp}\right|_{A^{c} \cup\left\{j, j^{\prime}\right\}}$ has rank $n-r$. This also shows that $v_{j^{\prime}}^{\perp}$ is dependent on the remaining columns of $\left.M^{\perp}\right|_{A^{c} \cup\left\{j, j^{\prime}\right\}}$, so that (6) will at least have $b_{j^{\prime}} \neq 0$.

Now a dependence (5) extends by zeroes to a vector $\left(c_{1}, \ldots, c_{r+1}, 0, \ldots, 0\right)$ in $\mathbb{F}^{n}$ that lies in $\operatorname{ker}(M)$, and hence also lies in the row space of $M^{\perp}$. However, vectors in the row space of $M^{\perp}$ are covectors for $\left\{v_{\ell}^{\perp}\right\}$ in the sense that they give the values of linear functionals $f$ in $\left(\mathbb{F}^{n-r}\right)^{*}$ when applied to the list of vectors $\left(v_{1}^{\perp}, \ldots, v_{n}^{\perp}\right)$. Thus there is a functional $f$ having $f\left(v_{\ell}^{\perp}\right)=c_{\ell}$ for $\ell \in A$ and $f\left(v_{\ell}^{\perp}\right)=0$ for $\ell \in A^{c}$. Applying this $f$ to (6) gives $c_{j} b_{j}+c_{j^{\prime}} b_{j^{\prime}}=0$ which is equivalent to the remaining assertion of the proposition.

\section{Simplicial SPANNing tReES}

For a collection of subsets $S$ of some vertex set $V$, let $\langle S\rangle$ denote the (abstract) simplicial complex $S$ on $V$ generated by $S$, that is, $\langle S\rangle \subset 2^{V}$ consists of all subsets of $V$ contained in at least one subset from $S$. We recall the notion of a simplicial spanning tree in $S$, following Adin [1, Duval, Klivans and Martin [8, Kalai [14, and Maxwell [20].

Definition 9. Let $S$ be the collection of facets of a pure $k$-dimensional (abstract) simplicial complex. Say that $R \subset S$ is an $S$-spanning tree if

(i) $\langle R\rangle$ contains the entire $(k-1)$-skeleton of $\langle S\rangle$,

(ii) $\tilde{H}_{k}(\langle R\rangle ; \mathbb{Z})=0$, and

(iii) $\tilde{H}_{k-1}(\langle R\rangle ; \mathbb{Z})$ is finite.

We point out here three well-known features of this definition. 
Proposition 10. Fix the collection of facets $S$ of a pure $k$-dimensional simplicial complex.

(i) Condition (i) in Definition 9 is equivalent to $\tilde{H}_{k}(\langle S\rangle,\langle R\rangle ; \mathbb{Z})=\mathbb{Z}^{|S \backslash R|}$.

(ii) Condition (ii) in Definition 9 is equivalent to $\tilde{H}_{k}(\langle R\rangle ; \mathbb{Q})=0$.

(iii) All $S$-spanning trees $R$ have the same cardinality, namely

$$
|R|=|S|-\operatorname{rank}_{\mathbb{Z}} \tilde{H}_{k}(\langle S\rangle ; \mathbb{Z}) .
$$

Proof. Proof of (i). Note that $\tilde{H}_{k}(\langle S\rangle,\langle R\rangle ; \mathbb{Z})=\tilde{Z}_{k}(\langle S\rangle,\langle R\rangle ; \mathbb{Z})$, since $\langle S\rangle$ is $k$ dimensional. By definition, the relative cycle group $\tilde{Z}_{k}(\langle S\rangle,\langle R\rangle ; \mathbb{Z})$ equals the kernel of the map

$$
\partial_{k}: \tilde{C}_{k}(\langle S\rangle,\langle R\rangle ; \mathbb{Z}) \rightarrow \tilde{C}_{k-1}(\langle S\rangle,\langle R\rangle ; \mathbb{Z}) .
$$

Because both the source and target of $\partial_{k}$ are free abelian, with the source of rank $|S \backslash R|$, its kernel is free abelian of the same rank if and only if $\partial_{k}$ is the zero map. This last condition is equivalent to Condition (i) in Definition 9 .

Proof of (ii). This follows from the fact that $\langle R\rangle$ is $k$-dimensional, so that

$$
\begin{aligned}
\tilde{H}_{k}(\langle R\rangle ; \mathbb{Z}) & =\tilde{Z}_{k}(\langle R\rangle ; \mathbb{Z}), \text { and } \\
\tilde{H}_{k}(\langle R\rangle ; \mathbb{Q}) & =\tilde{Z}_{k}(\langle R\rangle ; \mathbb{Q}) .
\end{aligned}
$$

The cycle group $\tilde{Z}_{k}(\langle R\rangle ; \mathbb{Z})$ (respectively, $\tilde{Z}_{k}(\langle R\rangle ; \mathbb{Q})$ ) vanishes if and only if the collection of boundaries of simplices in $R$ are linearly independent over $\mathbb{Z}$ (respectively, over $\mathbb{Q}$ ). However, these two notions of linear independence are equivalent.

Proof of (iii). Consider this portion of the long exact sequence of the pair $(\langle S\rangle,\langle R\rangle)$

$$
\begin{array}{ccccc}
\tilde{H}_{k}(\langle R\rangle ; \mathbb{Z}) & \rightarrow & \tilde{H}_{k}(\langle S\rangle ; \mathbb{Z}) & \rightarrow & \tilde{H}_{k}(\langle S\rangle,\langle R\rangle ; \mathbb{Z}) \\
\| & \rightarrow & \tilde{H}_{k-1}(\langle R\rangle ; \mathbb{Z}) \\
0 & & \mathbb{Z}^{|S \backslash R|} & &
\end{array}
$$

Here the two vertical equalities come from Condition (ii) in Definition 9 and from assertion (i) above, respectively. Since the last term $\tilde{H}_{k-1}(\langle R\rangle ; \mathbb{Z})$ in this sequence is a finite abelian group by Condition (iii) in Definition 9, the sequence shows that $\tilde{H}_{k}(\langle S\rangle ; \mathbb{Z})$ is a subgroup of $\mathbb{Z}^{|S \backslash R|}$ of finite index. Consequently, it must be free abelian of the same rank. Hence $|S \backslash R|=\operatorname{rank}_{\mathbb{Z}} \tilde{H}_{k}(\langle S\rangle ; \mathbb{Z})$ and equation (7) follows.

The following observation essentially goes back to work of Kalai [14, Lemma 2].

Proposition 11. Fix a vertex set $V$ and a collection of $k$-dimensional simplices $S$. Consider a collection of $(k+1)$-dimensional faces $T$ of cardinality

$$
|T|:=\operatorname{rank}_{\mathbb{Z}} \tilde{H}_{k}(\langle S\rangle ; \mathbb{Z})
$$

for which $T \cup\langle S\rangle$ forms a simplicial complex $K$, that is, all boundaries of faces in $T$ lie in $\langle S\rangle$.

Then the following two assertions hold for any choice of an $S$-spanning tree $R$.

(i) The $|T| \times|T|$ matrix $\partial$ that represents the relative simplicial boundary map

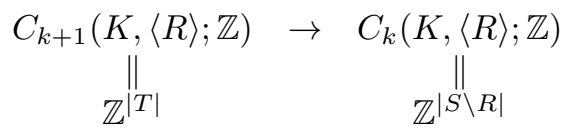

is nonsingular if and only if $\tilde{H}_{k+1}(K ; \mathbb{Q})=0$. 
(ii) When the matrix $\partial$ is nonsingular, then $\operatorname{coker}(\partial)=\tilde{H}_{k}(K,\langle R\rangle ; \mathbb{Z})$.

Proof. Proof of (i). Start by noting that $\tilde{H}_{k}(\langle R\rangle ; \mathbb{Q})=0$ due to part (ii) of Proposition 10, Since $\langle R\rangle$ is only $k$-dimensional, it also has $\tilde{H}_{k+1}(\langle R\rangle ; \mathbb{Q})=0$. Consequently,

$$
\tilde{H}_{k+1}(K ; \mathbb{Q}) \cong \tilde{H}_{k+1}(K,\langle R\rangle ; \mathbb{Q})=Z_{k+1}(K,\langle R\rangle ; \mathbb{Q}) .
$$

where the isomorphism comes from the long exact sequence in homology for the pair $(K,\langle R\rangle)$, and the equality follows since $K$ is $(k+1)$-dimensional. Therefore $\tilde{H}_{k+1}(K ; \mathbb{Q})$ vanishes if and only if $Z_{k+1}(K,\langle R\rangle ; \mathbb{Q})$ vanishes, which occurs if and only the square matrix $\partial$ has vanishing kernel, i.e. it is nonsingular.

Proof of (ii). We first note that $C_{k}(K,\langle R\rangle ; \mathbb{Z})=Z_{k}(K,\langle R\rangle ; \mathbb{Z})$ due to these facts:

(a) every $k$-simplex in $K$ actually lies in $S$ by our assumption on $T$, and

(b) every boundary of a $k$-simplex in $S$ lies in $R$ by Condition (i) in Definition 9 . Thus when $\partial$ is nonsingular, one has

$$
\begin{aligned}
\operatorname{coker}(\partial) & =C_{k}(K,\langle R\rangle ; \mathbb{Z}) / B_{k}(K,\langle R\rangle ; \mathbb{Z}) \\
& =Z_{k}(K,\langle R\rangle ; \mathbb{Z}) / B_{k}(K,\langle R\rangle ; \mathbb{Z})=\tilde{H}_{k}(K,\langle R\rangle ; \mathbb{Z}) .
\end{aligned}
$$

Definition 12. Given a collection of $k$-simplices $S$, and an $S$-spanning tree $R$, say ${ }^{2}$ that $R$ is torsion-free if Condition (iii) in Definition 9 is strengthened to the vanishing condition

(iv) $\tilde{H}_{k-1}(\langle R\rangle ; \mathbb{Z})=0$.

Example 13. For example, when $\langle R\rangle$ is a contractible subcomplex of $\langle S\rangle$ then it satisfies Condition (ii) of Definition 9 as well as the vanishing condition (iv). If it furthermore satisfies Condition (i) of Definition [9, then $R$ becomes a torsion-free $S$-spanning tree.

A frequent combinatorial setting where this occurs (such as in Proposition 15 below) is when $S$ is the set of facets of a (pure) shellable [3] simplicial complex, and $R$ is the subset of facets which are not fully attached along their entire boundaries during the shelling process.

Proposition 14. Using the hypotheses and notation of Proposition 11, if one assumes in addition that $R$ is torsion-free, assertion (ii) of Proposition 11 becomes the following assertion about (non-relative) homology:

(ii) When the matrix $\partial$ is nonsingular, then $\operatorname{coker}(\partial)=\tilde{H}_{k}(K ; \mathbb{Z})$

Proof. When $R$ is torsion-free, the long exact sequence for the pair $(K,\langle R\rangle)$ shows that $\tilde{H}_{k}(K ; \mathbb{Z}) \cong \tilde{H}_{k}(K,\langle R\rangle ; \mathbb{Z})$.

\section{More on the Complete $d$-PARtite Complex}

It is well-known and easy to see that for a positive integer $n$ having prime factorization $n=p_{1}^{e_{1}} \cdots p_{d}^{e_{d}}$ with $e_{i} \geq 1$, one has $\Phi_{n}(x)=\Phi_{p_{1} \cdots p_{d}}\left(x^{n / p_{1} \cdots p_{d}}\right)$. Thus it suffices to interpret the coefficients of cyclotomic polynomials for squarefree $n$.

\footnotetext{
${ }^{2}$ This condition on an $S$-spanning tree also plays an important role in forthcoming work by Duval, Klivans and Martin [9].
} 
In this section, we fix such a squarefree $n=p_{1} \cdots p_{d}$, and discuss further properties of the simplicial complexes $K_{p_{1}, \ldots, p_{d}}$, defined in Section 1, appearing in Theorems 1 and 2 .

Proposition 15. The $(d-2)$-dimensional skeleton of $K_{p_{1}, \ldots, p_{d}}$ is shellable, with

$$
\tilde{H}_{d-2}\left(K_{p_{1}, \ldots, p_{d}} ; \mathbb{Z}\right)=\mathbb{Z}^{n-\phi(n)} .
$$

Proof. To show that the $(d-2)$-skeleton is shellable, we note the following three facts: (i) zero-dimensional complexes are all trivially shellable, (ii) joins of shellable complexes are shellable [25, Sec. 2], and (iii) skeleta of (pure) shellable simplicial complexes are shellable [5, Corollary 10.12]. Having shown that this skeleton is shellable, it therefore has only top homology; see, for example [3, Appendix]. This homology is free abelian, of rank equal to the absolute value of its reduced Euler characteristic, namely

$$
\begin{aligned}
\left|\sum_{i \geq-1}(-1)^{i} \operatorname{rank}_{\mathbb{Z}}\left(C_{i}\right)\right| & =\left|\sum_{i \geq-1}(-1)^{i} \sum_{\substack{I \subsetneq\{1,2, \ldots, d\} \\
|I|=i+1}} \prod_{i \in I} p_{i}\right| \\
& =\left|\sum_{I \subsetneq\{1,2, \ldots, d\}}(-1)^{|I|-1} \prod_{i \in I} p_{i}\right| \\
& =\left|\left(p_{1}-1\right) \cdots\left(p_{d}-1\right)-p_{1} \cdots p_{d}\right| \\
& =|\phi(n)-n| .
\end{aligned}
$$

As noted in the introduction, the Chinese Remainder Theorem isomorphism (2) identifies elements of $\mathbb{Z} / n \mathbb{Z}$ with the $(d-1)$-dimensional simplices of $K_{p_{1}, \ldots, p_{d}}$. Lower dimensional faces of $K_{p_{1}, \ldots, p_{d}}$ can also be identified as cosets of subgroups within $\mathbb{Z} / n \mathbb{Z}$, but we will use this identification sparingly in this paper. For the sake of writing down oriented simplicial boundary maps, choose the following orientation on the simplices of $K_{p_{1}, \ldots, p_{d}}$, consistent with the orientation of facets preceding Theorem 2, choose the oriented $(\ell-1)$-simplex $\left[j_{i_{1}} \bmod p_{i_{1}}, \ldots, j_{i_{\ell}} \bmod p_{i_{\ell}}\right]$ with $i_{1}<\ldots<i_{\ell}$ as a basis element of $C_{\ell-1}\left(K_{p_{1}, \ldots, p_{d}} ; \mathbb{Z}\right)$. The following simple observation was the crux of the results in [19].

Proposition 16. If one identifies the indexing set $\mathbb{Z} / n \mathbb{Z}$ for the columns of the boundary map

$$
C_{d-1}\left(K_{p_{1}, \ldots, p_{d}} ; \mathbb{Z}\right) \rightarrow C_{d-2}\left(K_{p_{1}, \ldots, p_{d}} ; \mathbb{Z}\right)
$$

with the set $\mu_{n}:=\left\{\zeta^{j}\right\}_{j \in \mathbb{Z} / n \mathbb{Z}}$ of all $n^{\text {th }}$ roots of unity, then every row of this boundary map represents a $\mathbb{Q}$-linear dependence on $\mu_{n}$.

Proof. A row in this boundary map is indexed by an oriented $(d-2)$-face, which has the form $\left[j_{1} \bmod p_{1}, \ldots, j_{k} \widehat{\bmod } p_{k}, \ldots, j_{d} \bmod p_{d}\right]$ for some $j_{k} \in\left\{0,1, \ldots, p_{k}-1\right\}$ and $1 \leq k \leq d$. This row will then contain all zeroes except for entries equal to 
$(-1)^{k-1}$ in the columns indexed by $\zeta^{j}$ where

$$
\begin{gathered}
j \equiv j_{1} \bmod p_{1}, \\
\vdots \\
j \equiv j_{d} \bmod p_{d}
\end{gathered}
$$

except that $j \bmod p_{k}$ is allowed to be arbitrary. These exponents $j$ are exactly those lying in one coset of the subgroup $p_{1} \cdots \hat{p_{k}} \cdots p_{d} \mathbb{Z} / n \mathbb{Z}$ within $\mathbb{Z} / n \mathbb{Z}$. Summing $\zeta^{j}$ over $j$ in such a coset gives zero.

Example 17. Let $n=15$ as in Example 3, and consider the matrix for the simplicial boundary map $C_{1}\left(K_{3,5} ; \mathbb{Z}\right) \rightarrow C_{0}\left(K_{3,5} ; \mathbb{Z}\right)$. One of its rows is indexed by the 0 -face $[2 \bmod 5]$ and this row has exactly three nonzero entries, all equal to $(-1)^{0}=+1$. To see these signs, we rewrite $[2 \bmod 5]$ in three ways, all of which involve deleting the first entry out of two in an oriented 1-face:

$$
[2 \bmod 5]=[0 \widehat{\bmod } 3,2 \bmod 5]=[1 \widehat{\bmod } 3,2 \bmod 5]=[2 \widehat{\bmod } 3,2 \bmod 5] .
$$

The columns corresponding to these three 1-faces are indexed by the roots of unity $\zeta^{12}, \zeta^{7}$, and $\zeta^{2}$, respectively. Summing these up with coefficients of positive one, we get

$$
1 \cdot \zeta^{12}+1 \cdot \zeta^{7}+1 \cdot \zeta^{2}=\zeta^{2}\left(\zeta^{10}+\zeta^{5}+1\right)
$$

which is the sum of $\zeta^{j}$ over $j$ lying in a coset of $5 \mathbb{Z} / 15 \mathbb{Z}$, and hence is zero.

Definition 18. Assume that $n$ is squarefree and let $T$ denote any set of $n-\phi(n)$ columns of the boundary map (8). Identify the complementary set $T^{c}$ of $\phi(n)$ columns with a subset of the $n^{\text {th }}$ roots-of-unity $\mu_{n}$. Create a subcomplex of $K_{p_{1}, \ldots, p_{d}}$ by including its entire $(d-2)$-skeleton and attaching the subset of $(d-1)$-faces indexed by $T$. We denote this subcomplex as $K[T]$.

Recall from the introduction that for any subset $A \subseteq\{0,1, \ldots, \phi(n)\} \subset \mathbb{Z} / n \mathbb{Z}$, we let $K_{A}$ denote the subcomplex of $K_{p_{1}, \ldots, p_{d}}$ generated by the facets $\left\{F_{j \bmod n}\right\}$ as $j$ runs through the set of residues $A \sqcup A_{0}$ where

$$
A_{0}:=\{\phi(n)+1, \phi(n)+2, \ldots, n-2, n-1\} .
$$

Proposition 19. The subcomplex $K_{\varnothing}$, and hence every subcomplex $K_{A}$, contains the full $(d-2)$-skeleton of $K_{p_{1}, \ldots, p_{d}}$. Consequently, $K_{A}=K\left[A \sqcup A_{0}\right]$.

Proof. Since a $(d-2)$-face of $K_{p_{1}, \ldots, p_{d}}$ corresponds to a coset $j_{0}+\frac{n}{p_{i}}(\mathbb{Z} / n \mathbb{Z})$ within $\mathbb{Z} / n \mathbb{Z}$ for some $i=1,2, \ldots, d$ and $j_{0} \in \mathbb{Z} / n \mathbb{Z}$, one must check that every such coset intersects $A_{0}$. As $A_{0}$ is a consecutive sequence of $n-\phi(n)$ residues, this amounts to checking that

$$
n-\phi(n) \geq \frac{n}{p_{i}} \quad \text { for } \quad i=1,2, \ldots, d,
$$

or equivalently, that

$$
n\left(1-\frac{1}{p_{i}}\right) \geq \phi(n)=n\left(1-\frac{1}{p_{1}}\right) \cdots\left(1-\frac{1}{p_{d}}\right) .
$$

This inequality holds since each factor in parenthesis on the right is less than 1. 
We next point out an interesting feature of the labelling of the boundary map (8) with regard to the set $P_{n}$ of primitive $n^{\text {th }}$ roots of unity, noted already in [19, Remark 5]. Let $P_{n}^{c}$ denote the $(n-\phi(n))$-element subset of $\mu_{n}$ indexed by the $n^{\text {th }}$ roots of unity which are not primitive.

Proposition 20. Let $n$ be a squarefree integer and $P_{n}^{c}$ be as above. Then the subcomplex $K\left[P_{n}{ }^{c}\right]$ of $K_{p_{1}, \ldots, p_{d}}$ is contractible.

Proof. Observe that the primitive roots in $\mathbb{Z} / n \mathbb{Z}$ are exactly those elements which do not vanish modulo $p_{i}$ for $i=1, \ldots, d$. Tracing through the labelling of the $(d-1)$-faces via $\Xi$, we obtain the description

$$
K\left[P_{n}^{c}\right]=\bigcup_{i=1}^{d} \operatorname{star}_{K_{p_{1}, \ldots, p_{d}}}\left(0 \bmod p_{i}\right),
$$

where $\operatorname{star}_{\Delta}(v)$ denotes the simplicial star of the vertex $v$ inside a simplicial complex $\Delta$. Furthermore, each intersection of these stars is nonempty and contractible, because it is the star of another face: for $I \subset[d]$,

$$
\bigcap_{i \in I} \operatorname{star}_{K_{p_{1}, \ldots, p_{d}}}\left(0 \bmod p_{i}\right)=\operatorname{star}_{K_{p_{1}, \ldots, p_{d}}}\left(\left\{0 \bmod p_{i}\right\}_{i \in I}\right) .
$$

A standard nerve lemma [4, Theorem 10.6] then shows that $K\left[P_{n}{ }^{c}\right]$ itself is contractible. This also follows by induction on $d$, where the case $d=2$ appears as 12 , Exercise 0.23, p. 20].

Theorem 21. Let $n$ be a squarefree integer and $T$ be a subset of $\mu_{n}$ of size $n-\phi(n)$. Let $K[T]$ be the subcomplex of $K_{p_{1}, \ldots, p_{d}}$ of Definition [18, Then

$$
\tilde{H}_{i}(K[T] ; \mathbb{Z}) \cong \begin{cases}\mathbb{Z}[\zeta] / \mathbb{Z} T^{c} & \text { if } i=d-2, \\ \mathbb{Z} & \text { if } i=d-1 \text { and } \operatorname{rank}_{\mathbb{Z}}\left(\mathbb{Z} T^{c}\right)=\phi(n)-1, \\ 0 & \text { if } i=d-1 \text { and } \operatorname{rank}_{\mathbb{Z}}\left(\mathbb{Z} T^{c}\right)=\phi(n), \\ 0 & \text { if } i<d-2 .\end{cases}
$$

where $\mathbb{Z} T^{c}$ is the sublattice $\mathbb{Z}$-spanned by the roots-of-unity $T^{c} \subset \mu_{n}$.

Proof. Choose any $\mathbb{Z}$-basis for $\mathbb{Z}[\zeta]$. Let $M$ in $\mathbb{Z}^{\phi(n) \times n}$ be the matrix that expresses the $n^{\text {th }}$ roots of unity $\mu_{n}$ in this basis.

We construct a particular matrix $M^{\perp}$ to accompany $M$ as in Proposition 7 part (ii). Consider the collection $S$ of all $(d-2)$-faces in the complete $d$-partite complex $K_{p_{1}, \ldots, p_{d}}$. The complex $\langle S\rangle$ generated by $S$ is therefore the $(d-2)$ skeleton of $K_{p_{1}, \ldots, p_{d}}$. Proposition 15 implies that $\langle S\rangle$ is shellable, and that it has $\operatorname{rank}_{\mathbb{Z}} \tilde{H}_{d-2}(\langle S\rangle ; \mathbb{Z})=n-\phi(n)$. Therefore, we are in the situation of Example 13 implying that there exists a torsion-free $S$-spanning tree $R$, and any such $R$ will have $|S \backslash R|=n-\phi(n)$.

Our candidate for the matrix $M^{\perp}$ in $\mathbb{Z}^{(n-\phi(n)) \times n}$ is the restriction of the boundary map from (8) to its rows indexed by $S \backslash R$. Proposition [16 shows that the rows of $M^{\perp}$ are all perpendicular to the rows of $M$.

Now choose $T_{0}$ so that $T_{0}^{c}$ indexes the set $P_{n}$ of primitive $n^{\text {th }}$ roots of unity. Proposition [6implies that the maximal minor $\left.M\right|_{T_{0}^{c}}$ of $M$ is invertible over $\mathbb{Z}$, while Proposition 20 implies that the maximal minor $\left.M^{\perp}\right|_{T_{0}}$ of $M^{\perp}$ is invertible over $\mathbb{Z}$. Thus $M, M^{\perp}$ satisfy the hypotheses of Proposition 7 part (ii), and combining this with Proposition 14 gives the assertion of the theorem. 
Remark 22. Note that Theorem 21] makes no assertion about $\tilde{H}_{d-1}(K[T] ; \mathbb{Z})$ when $\operatorname{rank}_{\mathbb{Z}}\left(\mathbb{Z} T^{c}\right) \leq \phi(n)-2$, as these cases will not be needed in the proofs of Theorem 1 and 2. The authors thank Giacomo Condrò for pointing out that this result was inadvertently misstated in previous versions of this paper, including the journal version.

\section{Proof of Theorems 1 and 2}

We are now in a position to prove Theorems 1 and 2

Proof of Theorem 1, Let $T^{c}=\left\{1, \zeta, \zeta^{2}, \ldots, \zeta^{\phi(n)}\right\} \backslash\left\{\zeta^{j}\right\}$, so that $T=A_{0} \cup\{j\}$, and $K[T]=K\left[A_{0} \cup\{j\}\right]=K_{\{j\}}$ by Proposition 19, Note that $\operatorname{rank}_{\mathbb{Z}}\left(\mathbb{Z} T^{c}\right)$ is either $\phi(n)$ or $\phi(n)-1$, since $T^{c}=\left(\left\{1, \zeta, \zeta^{2}, \ldots, \zeta^{\phi(n-1)}\right\} \cup\left\{\zeta^{\phi(n)}\right\}\right) \backslash\left\{\zeta^{j}\right\}$, and

$$
\operatorname{rank}_{\mathbb{Z}}\left(\mathbb{Z}\left\{1, \zeta, \zeta^{2}, \ldots, \zeta^{\phi(n)-1}\right\}\right)=\operatorname{rank}_{\mathbb{Z}}[\mathbb{Z}[\zeta]]=\phi(n) .
$$

The theorem then follows from Theorem 21 and Corollary 5 .

Proof of Theorem . We prove Theorem 2 by applying Proposition 8 to the matrices $M, M^{\perp}$ in the proof of Theorem 21, with $A=\left\{1, \zeta, \zeta^{2}, \ldots, \zeta^{\phi(n)}\right\}$. Thus $A^{c}=A_{0}$ and $K_{\left\{j, j^{\prime}\right\}}=K\left[A^{c} \cup\left\{j, j^{\prime}\right\}\right]$ by Proposition 19.

The dependence (5) among the columns of $\left.M\right|_{A}$ has the same coefficients (up to scaling) as the cyclotomic polynomial, and the dependence (6) among the columns of $\left.M^{\perp}\right|_{A^{c} \cup\left\{j, j^{\prime}\right\}}$ will have the same coefficients (up to scaling) as a nonzero cycle $z=\sum_{\ell} b_{\ell}\left[F_{\ell}\right]$ in $\tilde{H}_{d-1}\left(K_{\left\{j, j^{\prime}\right\}} ; \mathbb{Z}\right)$.

\section{Attaching MAP REFORMUlation}

The authors are indebted to Dmitry Fuchs for suggesting reformulations of Theorems 1 and 2. explaining how the complete $d$-partite complex $K_{p_{1}, \ldots, p_{d}}$ is built from its subcomplex $K_{\varnothing}$, by attaching the facets $F_{j \bmod n}$ along their boundaries. We briefly discuss this here, beginning with a homological version. Throughout this section, all homology groups are reduced, and taken with coefficients in $\mathbb{Z}$.

Recall from the Introduction that for $j$ in $\mathbb{Z} / n \mathbb{Z}$, the facet $F_{j \bmod n}$ was given a particular orientation $\left[F_{j \bmod n}\right]$ as a basis element in the oriented reduced $(d-1)$ chains of $K_{p_{1}, \ldots, p_{d}}$. Let $\left[z_{j \bmod n}\right]:=\partial\left[F_{j \bmod n}\right]$ denote the $(d-2)$-cycle which is its image under the simplicial boundary map $\partial$.

Letting $\mathbb{S}^{d}, \mathbb{B}^{d}$ denote the $d$-dimensional sphere and ball respectively, denote by $\mathbb{S}^{d-1} \cup \mathbb{B}_{f}$ the space obtained by attaching $\mathbb{B}^{d}$ to $\mathbb{S}^{d-1}$ along its boundary $\operatorname{Bd}\left(\mathbb{B}^{d}\right)$ via a $\operatorname{map} \operatorname{Bd}\left(\mathbb{B}^{d}\right) \stackrel{f}{\rightarrow} \mathbb{S}^{d-1}$. Recall (see, e.g. [12, pp. 12-13, §2.2, and Cor. 4.25]) that the homotopy type of $\mathbb{S}^{d-1} \cup_{f} \mathbb{B}^{d}$ is determined by the absolute value of $\operatorname{deg}(f)$, the scalar defined by the map on the top homology groups

$$
\tilde{H}_{d-1}\left(\operatorname{Bd}\left(\mathbb{B}^{d}\right)\right) \cong \mathbb{Z} \stackrel{f_{*}}{\longrightarrow} \mathbb{Z} \cong \tilde{H}_{d-1}\left(\mathbb{S}^{d}\right) .
$$

Theorem 23. Let $n=p_{1} \cdots p_{d}$ be squarefree.

(i) One has a homology isomorphism

$$
\tilde{H}_{*}\left(K_{\varnothing}\right) \cong \tilde{H}_{*}\left(\mathbb{S}^{d-2}\right),
$$

with $\tilde{H}_{d-2}\left(K_{\varnothing}\right) \cong \mathbb{Z}$ generated by the cycle $\left[z_{\phi(n) \bmod n}\right]$. 
(ii) If $\Phi_{n}(x)=\sum_{j=0}^{\phi(n)} c_{j} x^{j}$, then for $j=0,1, \ldots, \phi(n)$, one has

$$
\left[z_{j \bmod n}\right]=c_{j}\left[z_{\phi(n) \bmod n}\right] \quad \text { in } \quad \tilde{H}_{d-2}\left(K_{\varnothing}\right) \cong \mathbb{Z}
$$

and a homology isomorphism

$$
\tilde{H}_{*}\left(K_{\{j\}}\right) \cong \tilde{H}_{*}\left(\mathbb{B}^{d-1} \cup_{f_{j}} \mathbb{S}^{d-2}\right)
$$

where $\operatorname{deg}\left(f_{j}\right)=c_{j}$.

Proof. Proposition 19] shows that all of the spaces $K_{A}$ share the same $(d-2)$-skeleton as $K_{p_{1}, \ldots, p_{d}}$, and hence they share the same homology groups $\tilde{H}_{i}$ for $i<d-2$. Furthermore, this $(d-2)$-skeleton was shown in Proposition [15] to be shellable, with top cycle/homology group $\tilde{Z}_{d-2} \cong \mathbb{Z}^{n-\phi(n)}$. Thus the $i$-dimensional homology groups with $i<d-2$ for any $K_{A}$ will vanish, in agreement with the homology of $\mathbb{S}^{d-1}$ and $\mathbb{S}^{d-1} \cup_{f_{j}} \mathbb{B}^{d}$.

It only remains to show the various assertions within (i) and (ii) for $(d-1)$ - and $(d-2)$-homology. Note that the complex $K_{\{\phi(n)\}}$ is $\mathbb{Z}$-acyclic, by Theorem 1 and the fact that $c_{\phi(n)}=+1$ since $\Phi_{n}(x)$ is monic of degree $\phi(n)$. Since $K_{\{\phi(n)\}}$ also has exactly $n-\phi(n)$ facets $\left\{F_{j \bmod n}\right\}_{j=\phi(n)}^{n-1}$, their boundary cycles $\left[z_{j \bmod n}\right]$ must form a $\mathbb{Z}$-basis for the $(d-2)$-cycle lattice $\tilde{Z}_{d-2} \cong \mathbb{Z}^{n-\phi(n)}$. Since the subcomplex $K_{\varnothing}$ of $K_{\{\phi(n)\}}$ has the same $(d-2)$-skeleton and contains all of its facets except for $F_{\phi(n) \bmod n}$, the assertions of (i) follow.

By assertion (i), for any $j=0,1, \ldots, \phi(n)-1$, there will be a unique integer $c$ for which $\left[z_{j \bmod n}\right]=c\left[z_{\phi(n) \bmod n}\right]$ in $\tilde{H}_{d-2}\left(K_{\varnothing}\right) \cong \mathbb{Z}$. This is equivalent to the assertion that there is a $(d-1)$-cycle in $K_{\{j, \phi(n)\}}$ of the form

$$
\left[F_{j \bmod n}\right]-c\left[F_{\phi(n) \bmod n}\right]+\sum_{\ell=\phi(n)+1}^{n-1} b_{\ell}\left[F_{\ell \bmod n}\right] .
$$

Taking $j^{\prime}=\phi(n)$ in Theorem 2, and bearing in mind that $c_{\phi(n)}=+1$, this forces $c=c_{j}$, as asserted in (ii).

Lastly, note that $K_{\{j\}}$ shares the same $(d-2)$-skeleton as $K_{\{\phi(n)\}}$, and shares most of the same facets, except for replacing the facet $F_{\phi(n) \bmod n}$ with $F_{j \bmod n}$. This means that the $(d-2)$-boundaries of $K_{\{j\}}$ will span the sublattice of the $(d-2)$-cycle lattice $\tilde{Z}_{d-2} \cong \mathbb{Z}^{n-\phi(n)}$ in which the $\mathbb{Z}$-basis element $\left[z_{\phi(n) \bmod n}\right]$ is replaced by $\left[z_{j \bmod n}\right]=c_{j}\left[z_{\phi(n) \bmod n}\right]$. Thus the $(d-1)$-homology of $K_{\{j\}}$ still vanishes, and its $(d-2)$-homology is the quotient lattice $\mathbb{Z} / c_{j} \mathbb{Z}$. This agrees with $\tilde{H}_{i}\left(\mathbb{B}^{d-1} \cup_{f_{j}} \mathbb{S}^{d-2}\right)$ for $i=d-1, d-2$.

Note that Theorem 23 does not circumvent Theorems 1 and Theorems 2, It uses both in a crucial way, thereby relying ultimately on the matroid duality inherent in the proofs of these results.

Remark 24. D. Fuchs also suggested the following further reformulation of the main results.

Proposition 25. Define a $(d-1)$-cochain $b$ on the complete $d$-partite complex $K_{p_{1}, \ldots, p_{d}}$ whose value on $\left[F_{j \bmod n}\right]$ is $c_{j}$ for $j=0,1, \ldots, \phi(n)$, and 0 otherwise. Then $b$ is a coboundary.

Proof. Extend the $\mathbb{Z}$-basis $\left\{\left[z_{j} \bmod n\right]\right\}_{j=\phi(n)}^{n-1}$ for the $(d-2)$-cycles $\tilde{Z}_{d-2}$ to a $\mathbb{Z}$-basis for the $(d-2)$-chains $\tilde{C}_{d-2}$ of $K_{p_{1}, \ldots, p_{d}}$. Then let $g$ in $G L_{\mathbb{Z}}\left(\tilde{C}_{d-2}\right)$ send this new basis 
to the standard basis of oriented $(d-2)$-faces $[f]$, and denote by $f_{0}$ the $(d-2)$-face for which $\left[f_{0}\right]=g\left[z_{\phi(n) \bmod n}\right]$. Theorem $23($ ii) shows that for $j=0,1, \ldots, \phi(n)$, the coefficient of $\left[f_{0}\right]$ when expanding $\left[z_{j \bmod n}\right]$ in the above basis for $\tilde{Z}_{d-2}$ is $c_{j}$. Thus $g\left[z_{j \bmod n}\right]$ has coefficient $c_{j}$ on $\left[f_{0}\right]$. This show that the $(d-2)$-cochain $\left[f_{0}\right]^{*}$ dual to $\left[f_{0}\right]$ has the property that the coboundary map $\partial^{*}$ sends $g^{*}\left[f_{0}\right]^{*}$ to $b$ :

$$
\begin{aligned}
\partial^{*} g^{*}\left[f_{0}\right]^{*}\left(\left[F_{j \bmod n}\right]\right) & =\left[f_{0}\right]^{*}\left(g \partial\left[F_{j \bmod n}\right]\right)=\left[f_{0}\right]^{*}\left(g\left[z_{j \bmod n}\right]\right) \\
& = \begin{cases}c_{j} & \text { if } 0 \leq j \leq \phi(n) \\
0 & \text { if } \phi(n)+1 \leq j \leq n-1 .\end{cases}
\end{aligned}
$$

Question 26. Is there a natural choice of $a(d-2)$-chain having coboundary $b$ ? An affirmative answer would be helpful in writing down the coefficients of $\Phi_{n}(x)$.

We next give a homotopy-theoretic version of Theorem 23.

Theorem 27. For $d \geq 4$, and every $A \subseteq\{0,1, \ldots, \phi(n)\}$, the complex $K_{A}$ is simply-connected. Consequently, for $d \neq 3$, one has the following.

(i) The complex $K_{\varnothing}$ is homotopy equivalent to $\mathbb{S}^{d-2}$, and contains $\left[z_{\phi(n) \bmod n}\right]$ as a fundamental $(d-2)$-cycle.

(ii) For $j=0,1, \ldots, \phi(n)$, the cyclotomic polynomial coefficient $c_{j}$ gives the degree of the attaching map from the oriented boundary $\left[z_{j} \bmod n\right]$ of the facet $F_{j \bmod n}$ into the homotopy $(d-2)$-sphere $K_{\varnothing}$, with respect to the choice of $\left[z_{\phi(n) \bmod n}\right]$ as the fundamental cycle.

(iii) In particular, the complex $K_{\{j\}}$ is homotopy equivalent to $\mathbb{S}^{d-2} \cup_{f_{j}} \mathbb{B}^{d-1}$ where $\operatorname{deg}\left(f_{j}\right)=c_{j}$.

Proof. For $d=1,2$, the assertions (i),(ii),(iii) follow trivially from Theorem 23

When $d \geq 4$, first observe that the fundamental group of $K_{A}$ is determined by its 2-skeleton, which is the same as the 2-skeleton of $K_{p_{1}, \ldots, p_{d}}$, by Proposition 19 . The latter skeleton is shellable by Proposition 15, hence homotopy equivalent to a wedge of $(d-2)$-spheres, and therefore simply-connected.

For the remaining assertions when $d \geq 4$, since $K_{\varnothing}$ is simply-connected and has the homology of $\mathbb{S}^{d-2}$ by Theorem 23(i), assertion (i) follows from a standard application of the Hurewicz isomorphism theorem [26, Theorem 5 part (ii), p.398] and the homological Whitehead theorem [12, Cor. 4.33]. Assertion (ii) now follows from Theorem 23(ii), and assertion (iii) follows combining assertions (i) and (ii).

Question 28. Let $d=3$, so that $n=p_{1} p_{2} p_{3}$ for three distinct primes $p_{1}, p_{2}, p_{3}$.

- Is $K_{\varnothing}$ homotopically equivalent to the circle $\mathbb{S}^{1}$ ?

- Is $K_{\{j\}}$ homotopically equivalent to $\mathbb{B}^{2} \cup_{f_{j}} \mathbb{S}^{1}$, where $\operatorname{deg}\left(f_{j}\right)=c_{j}$, for $j=0,1, \ldots, \phi(n) ?$

One might hope, for example, to achieve such homotopy equivalences by a sequence of elementary collapses. However, in the example of $n=105=3 \cdot 5 \cdot 7$, with $\phi(n)=48$, a computer exploration indicated that

- $K_{\varnothing}$ did not collapse down to something homeomorphic to $\mathbb{S}^{1}$,

- the $\mathbb{Z}$-acyclic complexes $K_{\{0\}}, K_{\{48\}}$ did not collapse down to a point, and

- the complex $K_{\{7\}}$, which Theorem 23 predicts has the homology of a real projective plane $\mathbb{R} P^{2}$ since $c_{7}=-2$, did not collapse down to an $\mathbb{R} P^{2}$. 


\section{Concordance with properties of $\Phi_{n}(x)$}

Subtleties in the spaces $K_{\{j\}}$ make it not yet clear whether Theorems 1 and 2 will prove useful in approaching classical questions about the coefficients of $\Phi_{n}(x)$, e.g. as discussed in [2, 10, 11, 15, 31. Nevertheless, we briefly explain here how various well-known properties of $\Phi_{n}(x)$ manifest themselves topologically in the complexes $K_{\{j\}}$ and $K_{\left\{j, j^{\prime}\right\}}$ that appear in Theorems 1 and 2 .

8.1. Two prime factors and graphs. When $d=2$ so that $n=p_{1} p_{2}$ is the product of only two primes, the subcomplexes $K_{\{j\}}$ of $K_{p_{1}, p_{2}}$ are 1-dimensional, that is, graphs. Hence their $(d-2)$-dimensional homology is their 0 -dimensional homology, which is always torsion-free. It follows that the only nonzero coefficients of $\Phi_{p_{1} p_{2}}(x)$ are \pm 1 , agreeing with a well-known old observation of Migotti [22]. The explicit expansion of $\Phi_{p_{1} p_{2}}(x)$ is given in Elder [10], Lam and Leung [17, and Lenstra [18].

8.2. Coefficient symmetry and simplicial automorphisms. It is not hard to see from (1) that, for $n>1$, the cyclotomic polynomial $\Phi_{n}(x)=\sum_{j=0}^{\phi(n)} c_{j} x^{j}$ is palindromic, that is, $c_{j}=c_{\phi(n)-j}$. For squarefree $n=p_{1} \cdots p_{d}$, this coefficient symmetry is reflected in certain simplicial automorphisms of the complex $K_{p_{1}, \ldots, p_{d}}$ which we discuss here.

Note that any element of the product of symmetric groups $\mathfrak{S}_{p_{1}} \times \cdots \times \mathfrak{S}_{p_{d}}$ that separately permutes each of the vertex sets $K_{p_{1}}, \ldots, K_{p_{d}}$ gives rise to a simplicial automorphism of $K_{p_{1}, \ldots, p_{d}}$, with the property that it sends a positively oriented facet $\left[F_{j \bmod n}\right]$ as in (3) to another positively oriented facet. We focus on a subgroup of these automorphisms isomorphic to the dihedral group of order $2 n$

$$
D_{2 n}:=\left\langle s, r: s^{2}=r^{n}=1, \text { srs }=r^{-1}\right\rangle .
$$

Let $s, r$ act simultaneously on each vertex set $K_{p_{i}}$ as follows:

$$
\begin{aligned}
& j \bmod p_{i} \stackrel{s}{\longmapsto} \quad-j \bmod p_{i} \\
& j \bmod p_{i} \stackrel{r}{\longmapsto} j+1 \bmod p_{i} .
\end{aligned}
$$

This induces their action on oriented facets as follows:

$$
\begin{array}{ll}
{\left[F_{j \bmod n}\right] \stackrel{s}{\longmapsto}} & {\left[F_{-j \bmod n}\right]} \\
{\left[F_{j \bmod n}\right] \stackrel{r}{\longmapsto}} & {\left[F_{j+1} \bmod n\right] .}
\end{array}
$$

In particular, the element $t:=r^{\phi(n)} s$ will be an involution that swaps the oriented facets $\left[F_{j \bmod n}\right],\left[F_{\phi(n)-j \bmod n}\right]$ for each $j$, and hence maps the complex $K_{\{j\}}$ isomorphically onto the complex $K_{\{\phi(n)-j\}}$. This shows, via Theorem 1 , that $c_{j}= \pm c_{\phi(n)-j}$.

Furthermore, $t$ maps $K_{\{j, \phi(n)\}}$ isomorphically onto $K_{\{\phi(n)-j, 0\}}$. This shows via Theorem 2 that $c_{j}, c_{\phi(n)-j}$ must also have the same sign: their sign difference would have to be the same as the sign difference between the leading coefficient $c_{\phi(n)}(=+1)$ and the constant coefficient $c_{0}(=+1)$.

8.3. Cyclotomic polynomials for even $n$ and suspension. It is also wellknown, and follows from (1), that $\phi(2 n)=\phi(n)$ for $n$ odd, and that the two 
cyclotomic polynomials

$$
\Phi_{2 n}(x)=\sum_{j=0}^{\phi(n)} \hat{c}_{j} x^{j} \quad \text { and } \quad \Phi_{n}(x)=\sum_{j=0}^{\phi(n)} c_{j} x^{j}
$$

determine each other by $\Phi_{2 n}(x)=\Phi_{n}(-x)$. Equivalently, $\hat{c}_{j}=(-1)^{j} c_{j}$ for all $j$. When $n=p_{1} \cdots p_{d}$ is squarefree, this manifests itself topologically as follows.

The complex $\hat{K}:=K_{2, p_{1}, \ldots, p_{d}}$ relevant to $\Phi_{2 n}(x)$ is the two-point suspension $\Sigma K$ of the complex $K:=K_{p_{1}, \ldots, p_{d}}$ relevant to $\Phi_{n}(x)$. Here the two suspension vertices in $\hat{K}$ are labelled $(0 \bmod 2)$ and $(1 \bmod 2)$. This means that every facet $F_{\ell \bmod n}$ of the complex $K$ is contained in exactly two facets $\hat{F}_{\ell \bmod 2 n}$ and $\hat{F}_{\ell+n \bmod 2 n}$ of $\hat{K}$.

Now consider the two subcomplexes $\hat{K}_{\{j\}}, K_{\{j\}}$ of $\hat{K}, K$ whose homology torsion predict the two coefficients $\hat{c}_{j}, c_{j}$ up to sign. We claim that $\hat{K}_{\{j\}}$ contains the two-point suspension $\Sigma K_{\{j\}}$ as a deformation retract. Specifically, $\Sigma K_{\{j\}}$ is the subcomplex of $\hat{K}_{\{j\}}$ generated by the facets $\left\{\hat{F}_{\ell \bmod 2 n}\right\}$ as $\ell$ runs through

$\{j, n+j\} \cup\{\phi(n)+1, n+\phi(n)+1, \quad \phi(n)+2, n+\phi(n)+2, \quad \ldots, \quad n-1,2 n-1\}$. The collection $\mathcal{F}$ of facets of $\hat{K}_{\{j\}}$ lying outside the subcomplex $\Sigma K_{\{j\}}$ is given by $\left\{\hat{F}_{\ell+n \bmod 2 n}\right\}$ for $\ell$ in $\{0,1, \ldots, \phi(n)\} \backslash\{j\}$. Each such facet $\hat{F}_{\ell+n \text { mod } 2 n}$ in $\mathcal{F}$ has a free codimension one face, namely the facet $F_{\ell \bmod n}$ of $K_{\{j\}}$, because the facet $\hat{F}_{\ell \text { mod } 2 n}$ of $\hat{K}$ is absent from $\hat{K}_{\{j\}}$. Thus one can remove the facets in $\mathcal{F}$ from $\hat{K}_{\{j\}}$ via a sequence of elementary collapses, leaving the retract $\Sigma K_{\{j\}}$. This explains why $\hat{c}_{j}= \pm c_{j}$.

The exact sign relationship $\hat{c}_{j}=(-1)^{j} c_{j}$ comes from a similar relationship between the complexes $\hat{K}_{\left\{j, j^{\prime}\right\}}, K_{\left\{j, j^{\prime}\right\}}$ of $\hat{K}, K$ : the two-point suspension $\Sigma K_{\left\{j, j^{\prime}\right\}}$ is a deformation retract of $\hat{K}_{\left\{j, j^{\prime}\right\}}$. One must also analyze the relation between the orientations of facets in a $(d-2)$-cycle $z$ of $K_{\left\{j, j^{\prime}\right\}}$ versus the orientations of the analgous facets in the suspended $(d-1)$-cycle $\Sigma(z)$ of $\hat{K}_{\left\{j, j^{\prime}\right\}}$; we omit this detailed analysis.

\section{ACKNowledgements}

The authors wish to credit Jeremy Martin for the original observation that the cyclotomic matroid $\mu_{n}$ with $n=p_{1} p_{2}$ is cographic, as well as several helpful comments. They also thank Sam Elder, Nathan Kaplan, and Pieter Moree for helpful references on cyclotomic polynomials, Dmitry Fuchs for suggestions that led to Section 7 and two anonymous referees for helpful edits. They are very grateful to Giacomo Condrò for pointing out the error in previous versions of this paper noted in Remark 22.

\section{REFERENCES}

[1] R.M. Adin, Counting colorful multi-dimensional trees. Combinatorica 12 (1992), 247-260.

[2] G. Bachman, On the Coefficients of Cyclotomic Polynomials Memoirs of the American Mathematical Society 106, 1993.

[3] A. Björner, Shellable and Cohen-Macaulay partially ordered sets. Trans. Amer. Math. Soc. 260 (1980), no. 1, 159-183.

[4] A. Björner, Topological methods. Handbook of combinatorics, Vol. II, 1819-1872. Elsevier, Amsterdam, 1995

[5] A. Björner and M. Wachs, Shellable nonpure complexes and posets. II. Trans. Amer. Math. Soc. 349 (1997), no. 10, 3945-3975. 
[6] E.D. Bolker, Simplicial geometry and transportation polytopes. Trans. Amer. Math. Soc. 217 (1976), 121-142.

[7] H.H. Crapo and G.-C. Rota, On the foundations of combinatorial theory: Combinatorial geometries. Preliminary edition. The M.I.T. Press, Cambridge, Mass.-London, 1970.

[8] A.M. Duval, C.J. Klivans, and J.L Martin, Simplicial matrix-tree theorems. Trans. Amer. Math. Soc. 361 (2009), no. 11, 6073-6114.

[9] A.M. Duval, C.J. Klivans, and J.L Martin, personal communication, January 2011.

[10] S. Elder, Flat cyclotomic polynomials: a new approach, manuscript in preparation; see also http://www.coloradomath.org/handouts/flat_cyclotomic.pdf

[11] Y. Gallot and P. Moree, Neighboring ternary cyclotomic coefficients differ by at most one. $J$. Ramanujan Math. Soc. 24 (2009), no. 3, 235-248.

[12] A. Hatcher, Algebraic Topology. Cambridge University Press, Cambridge, 2002.

[13] K. Johnsen, Lineare Abhängigkeiten von Einheitswurzeln. Elem. Math. 40 (1985), 57-59.

[14] G. Kalai, Enumeration of $\mathbb{Q}$-acyclic simplicial complexes. Israel J. Math. 45 (1983), 337-351.

[15] N. Kaplan, Flat cyclotomic polynomials of order three. J. Number Theory 127 (2007), no. $1,118-126$.

[16] G. S. Kazandzidis, On the cyclotomic polynomial: Coefficients. Bull. Soc. Math. Gréce (N.S.) 4 (1963), no. 1, 1-11.

[17] T.Y. Lam and K.H. Leung, On the Cyclotomic Polynomial $\Phi_{p q}(X)$. Amer. Math. Monthly 103 (1996), 562-564.

[18] H.W. Lenstra, Vanishing sums of roots of unity, in: Proceedings, Bicentennial Congress Wiskundig Genootschap, Vrije Univ., Amsterdam, 1978, Part II, 1979, pp. 249-268

[19] J. Martin and V. Reiner, Cyclotomic and simplicial matroids. Israel J. Math. 150 (2005), $229-240$.

[20] M. Maxwell, Enumerating bases of self-dual matroids. J. Combin. Theory Ser. A 116 (2009), no. $2,351-378$.

[21] R. Meshulam, Homology of balanced complexes via the Fourier transform. J. Algebraic Combin. 35 (2012) 565-571.

[22] A. Migotti, Zur Theorie der Kreisteilungsgleichung. Sitzber. Math.-Naturwiss. Classe der Kaiser. Akad. der Wiss., Wien 87 (1883), 7-14.

[23] J.R. Munkres, Elements of algebraic topology. Addison-Wesley Publishing Company, Menlo Park, CA, 1984.

[24] J.G. Oxley, Matroid theory. Oxford Science Publications. The Clarendon Press, Oxford University Press, New York, 1992.

[25] J.S. Provan and L.J. Billera, Decompositions of simplicial complexes related to diameters of convex polyhedra. Math. Oper. Res. 5 (1980), no. 4, 576-594.

[26] E.H. Spanier, Algebraic topology. Springer-Verlag, New York-Berlin, 1981.

[27] D.J.A. Welsh, Matroid theory. London Math. Soc. Monographs 8. Academic Press, LondonNew York, 1976.

[28] N. White, Theory of matroids. Encyclopedia of Mathematics and its Applications 26. Cambridge University Press, Cambridge, 1986.

[29] N. White, Combinatorial geometries. Encyclopedia of Mathematics and its Applications 29. Cambridge University Press, Cambridge, 1987.

[30] N. White, Matroid applications. Encyclopedia of Mathematics and its Applications 40. Cambridge University Press, Cambridge, 1992.

[31] J. Zhao and X. Zhang, Coefficients of ternary cyclotomic polynomials. J. Number Theory 130 (2010), no. 10, 2223-2237. 55455

Gregg Musiker, School of Mathematics, University of Minnesota, Minneapolis, MN

Email address: musiker@math.umn.edu

Victor Reiner, School of Mathematics, University of Minnesota, Minneapolis, MN 55455

Email address: reiner@math.umn.edu 